\title{
Dielectric properties and molecular motions of liquid crystal molecules in 4-(2-methylbytyl)phenyl 4-(4-octylphenyl)benzoate liquid crystal having blue phase (CE8)
}

\author{
W. OTOWSKI*, G. LEWIŃSKA \\ Institute of Physics, Cracow University of Technology, Podchorążych 1, 30-084 Kraków, Poland
}

\begin{abstract}
Blue phase liquid crystals exhibit unique properties which are used in the new type of display. A blue-phase liquid crystal display was first presented commercially by Samsung in 2007. The blue-phase-three-color pixel display eliminates the need for color filters. This type of display uses blue-phase multi-component liquid crystal. Considering the one-component systems, it turns out that they are stable only in a very narrow range of temperatures between the isotropic and the chiral nematic phase (about $1 \mathrm{~K}$ ). In 2005, a wide temperature range BP multi-component system was reported by researchers from the University of Cambridge. There are still several unsolved problems left. One of them is chemical stability and reliability. Therefore, the knowledge of molecular dynamics of blue phase liquid crystal is a prerequisite for understanding of blue-phase multi-component system. Understanding the molecular dynamics of a single component liquid-crystalline blue phase system can facilitate the solution of these problems. We present the molecular dynamics investigation of 4-(2-methylbytyl)phenyl 4-(4-octylphenyl)benzoate (CE8), which may be a good candidate to form materials suitable for blue-phase liquid crystal displays.
\end{abstract}

Keywords: liquid crystal; blue phase; molecular dynamics; dielectric spectroscopy; correlation factor

(C) Wroclaw University of Technology.

\section{Introduction}

Liquid crystals are structures built up of anisotropic molecules. However, the open question is how the one anisotropic molecule (as a main structural element) may set in order giving a phase or sequence of different phases. In particular, the blue phase arrangement is the most interesting, especially for a new type of blue-phase liquid crystal display. On the other hand, the knowledge of the molecular dynamics of liquid crystals (assuming that molecular motion is under consideration) is essential to create a complete "image" of liquid crystals. Therefore, the investigation of the substance having a relatively simple chemical structure and phase sequence containing BP seems to be interesting.

The conclusive parameters limiting technical application of BPs are the narrow temperature

*E-mail: w_otowski@wp.pl range (about $1{ }^{\circ} \mathrm{C}$ ) and the high temperature at which it appears. In 2005, researchers from the University of Cambridge reported their discovery of a new multi-component blue-phase liquid crystal that remains stable over a wide temperature range of $16{ }^{\circ} \mathrm{C}$ to $60{ }^{\circ} \mathrm{C}$ [1]. This multi-component system may be used to switch the color of reflected light by applying an electric field to a material. And in turn, the substance could be used to produce three-color (red, green and blue) pixels for full-color displays [2]. In 2007, Samsung presented the first LCD "Blue Phase" display. Samsung's prototype was based on the polymer-stabilized blue phase (PSBP) liquid crystals described by Kikuchi and co-workers [3]. Unfortunately, the BP phase possessing materials, stabilized by polymers, have a very limited lifetime. This probably is the reason that the commercial production of the LCD based on BPs is not foreseen. An overview of the recent developments of this new technology, readers can find in the following references [4-7]. 
The blue phase occurs extremely rarely and is an interesting system for studying the properties of oriented molecule systems. Generally, blue phase systems have a regular threedimensional cubic structure. The competition between the chiral forces and a "double twist" structure leads to a stable network [8]. The double twist means a structure in which the director twists simultaneously about two independent directions. The order parameter exhibits three-dimensional orientational ordering with periods of up to $500 \mathrm{~nm}$ (so do selective Bragg reflections in the visible light wavelength range). Upon increasing the temperature from the chiral nematic to isotropic phase there are three stable blue phases labelled as BPI, BPII and BPIII. Blue phases I (BPI) and II (BPII) show a cubic symmetry. For BPI it is a bodycentred cubic symmetry, in which elementary cell size changes with temperature. BPII shows a simple (three-dimensional) cubic symmetry and there is no change in elementary cell size with temperature. BPIII, called blue fog, is almost macroscopically amorphous (short-range chiral positional ordering - a local cubic lattice).

Taking into account the dielectric spectroscopy we need to realize that this technique monitors macroscopic properties of a system, i.e. dielectric spectroscopy measures the collective response of a charge-free system to an applied electric field. The entire spectral density of the molecular motion comes from a bulk liquid crystal. Thus, in dielectric experiment one observes the motion of a "probe molecule". The dielectric "probe molecule" should not be considered as a real liquid crystal mesogen. Hence, one should always consider how to transform a "probe molecule" to a real mesogen [9].

In this paper we will concentrate on molecular description of the reorientation observed in an undisturbed regime. An undisturbed regime means a system without any external agent, like, e.g., electric field. To this end we decided to carry out investigation of a bulk sample without any bias voltage. Anyhow, the low-frequency dielectric measurements of CE8 as the functions of both a bias electric field and temperature have been done by Cava et al. [10]. They measured CE8 liquid crystal in chiral and racemic forms. The main conclusion was that they observed one Cole-Cole process. However, one should keep in mind that ferroelectric liquid crystal under bias voltage presents other structure.

\section{Material}

The liquid crystal 4-(2-methylbutyl) phenyl 4(4-octylphenyl) benzoate was synthesized in 70s and, since then, has been a subject of research. The substance was marked as: $8 \mathrm{SI}^{*} 2 \mathrm{M} 4 \mathrm{P} 8 \mathrm{BC}$ and CE8. Nowadays, CE8 abbreviation is used commonly. This material as well as its mixtures, are still under investigation.

The substance under study (CE8) was synthesized in the Institute of Chemistry of the Military University of Technology in Warsaw. The compound is chiral, with an asymmetric carbon atom. The length of the molecule is $27.1 \AA$ and the width is $5.0 \AA$. The calculation was performed with ChemSketch version CAChe/PM5 [11]. CE8 can exist in many different conformations. Two enantiomers $\left(E_{1}\right.$ and $\left.E_{2}\right)$ have been chosen to calculate HOF - "heat of formation". The energies are equal within the limits of error: $\operatorname{HOF}\left(E_{1}\right)-$ $486.38[\mathrm{~kJ} / \mathrm{mol}], \operatorname{HOF}\left(\mathrm{E}_{2}\right)-486.42[\mathrm{~kJ} / \mathrm{mol}] . \mathrm{In}$ Fig. 1 we present a conformation which probably is the most stable [11].

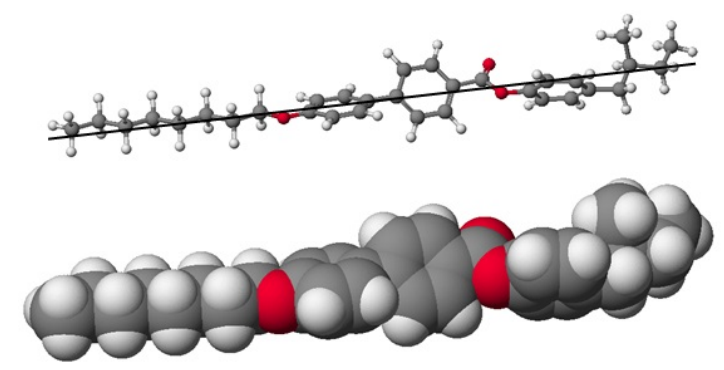

Fig. 1. A model of CE8 molecule

The total dipole moment of CE8 $\left(\mu_{\text {tot }}=2.27 \mathrm{D}\right)$ is almost parallel to the long molecular axis (the long molecular axis is the axis of the lowest moment of inertia): $\mu_{\mathrm{x}}=2.18 \mathrm{D}, \mu_{y}=0.50 \mathrm{D}$, $\mu_{\mathrm{z}}=0.36 \mathrm{D}$. It makes a relatively small angle 
$\beta=164.2^{\circ}$ with the long molecular axis (x-axis). The compound should have moderate positive dielectric anisotropy. The octyloxy chain is alltrans and in plane of the bond benzene ring. The benzene rings in the biphenyl moiety are twisted by $50.6^{\circ}$, which is the limit case reported in the literature about biphenyl. The carboxyl group - $\mathrm{COO}-$ is twisted by a dihedral angle of $30.8^{\circ}$. More exact calculations using ab initio methods yield about $0^{\circ}$ for this dihedral, which is close to experimental data in the solid state. However, ab initio methods cannot be applied for a large molecule, like the considered one. The right benzene ring is nearly perpendicular to the middle benzene ring. The butyl chain is in all-trans conformation, and is nearly perpendicular to the plane of the benzene ring, as usual in alkyl benzenes [11]. One may check our data comparing them with that given by Hoffmann for similar substance CE6 (Table 1) [12].

Kuczyński et al. [13] have identified six smectic phases, cholesteric phase and blue phases (they used a substance provided by British Drug Store and carried out measurements by optical methods and differential scanning calorimetry). Based on Xray diffraction studies, the Gierlotka's group [14] identified six smectic phases: SmG, SmJ, SmF, SmI, SmC, SmA. They used CE8 substance synthesized by the Dąbrowski's group [15]. Przedmojski examined CE8 twice $[16,17]$ and suggested the existence of six smectic phases, all of them chiral. Chirality of the smectic phases was also suggested by Budai et al. [18].

According to several publications, the substance was referred as ferroelectric liquid crystal. However, the latest publications by Kutnjak confirm the chirality of smectic C phase only [19]. Papers $[15,19-21]$ focused on ferroelectric properties of the substance.

In 1990, Drevensek et al. [22] presented the order parameter fluctuations in the transition phase between the smectic A and smectic $C^{*}$ phases. The results were obtained by correlated photons method.

The paper by Kuczyński concerned the helix size changes versus temperature in chiral smectic $\mathrm{C}^{*}[23]$.
The CE8 substance was used also as a base or a component of mixtures. As reported Kutnjak et al. [24], dispersed silica particles were added to CE8. The mixtures were examined by dielectric spectroscopy and calorimetry methods [25]. Rosenzweig described the optical activity of CE8 and CE8R (racemic) mixtures [26]. In [25], the results of tests carried out for a derivative of the substance (a mixture of CE8 and CdSe) were presented. The mixture exhibited a wide range blue phase.

The optical studies (polarized optical microscopy - POM) were performed, as well. The very first results were published by Lewińska et al. [27]. Further POM measurements showed the existence of four smectic phases [28].

\section{Experimental}

For the measured substance we observe four smectic phases, one cholesteric and one blue phase (stable within about 4 degrees). Based on texture images, the following identification of smectic phases is postulated: Sm1 mesophase (mosaic texture) as SmG, Sm2 mesophase (fun-shaped texture) as SmI, Sm3 mesophase (fun-shaped, fingerprint texture) as $\mathrm{SmC}$, and $\mathrm{Sm} 4$ (focal conic and fun-shaped texture) as SmA.

The textures of smectic I and smectic G expose a strong paramorphosis [28]. However, phase transitions between the phases are clearly visible. The blue phase texture is shown in Fig. 2.

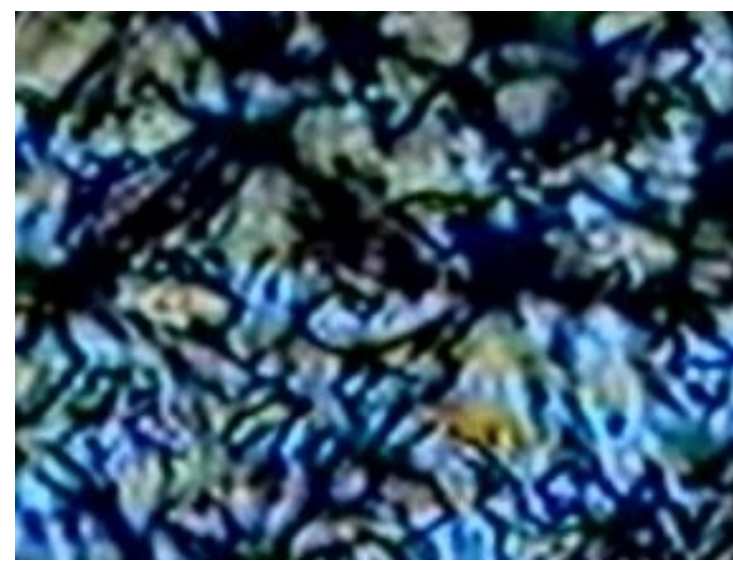

Fig. 2. Blue phase texture. 
Table 1. Values of the molecular parameters of the investigated liquid crystal.

\begin{tabular}{|c|c|c|c|c|c|c|c|c|c|c|}
\hline & $\begin{array}{c}\mathrm{HOF} \\
{[\mathrm{kJ} / \mathrm{mol}]}\end{array}$ & $\begin{array}{c}\mu_{\mathrm{x}} \\
{[\mathrm{D}]}\end{array}$ & $\begin{array}{c}\mu_{\mathrm{y}} \\
{[\mathrm{D}]}\end{array}$ & $\begin{array}{l}\mu_{\mathrm{z}} \\
{[\mathrm{D}]}\end{array}$ & $\begin{array}{l}\mu_{\text {tot }} \\
{[D]}\end{array}$ & $\begin{array}{c}\beta \\
{\left[{ }^{\circ}\right]} \\
\end{array}$ & $\begin{array}{c}\alpha_{\mathrm{x}} \\
{\left[10^{-41} \mathrm{Fm}^{2}\right]}\end{array}$ & $\begin{array}{c}\alpha_{\mathrm{y}} \\
{\left[10^{-41} \mathrm{Fm}^{2}\right]}\end{array}$ & $\begin{array}{c}\alpha_{\mathrm{z}} \\
{\left[10^{-41} \mathrm{Fm}^{2}\right]}\end{array}$ & $\begin{array}{c}\alpha \\
{\left[10^{-41} \mathrm{Fm}^{2}\right]}\end{array}$ \\
\hline$\overline{\mathrm{E}_{1}}$ & -486.38 & 2.18 & 0.50 & 0.36 & 2.27 & 164.2 & 943.0 & 447.3 & 328.1 & 572.8 \\
\hline $\mathrm{E}_{2}$ & -486.42 & 2.19 & 0.58 & 0.31 & 2.29 & 166 & 942.2 & 447.9 & 328.3 & 572.8 \\
\hline $\mathrm{CE}^{(1)}$ & & & & & 2.10 & 165 & & & & 555 \\
\hline
\end{tabular}

${ }^{(1)}$ data presented in [12].

TOA (thermo-optical analysis) measurements point out the existence of phase transitions between the crystalline phase and smectic phase [29]. Phase transitions between smectic phases and between smectic phase and cholesteric phase are visible [28]. During the cooling run TOA curve shows a phase transition to the blue phase.

After analyzing the data presented above we suggest the final phase diagram [27]:

- on heating: $\mathbf{C r} 49.2{ }^{\circ} \mathrm{C} \mathrm{SmG} 66.5{ }^{\circ} \mathrm{C} \mathrm{SmI}$ $68.9{ }^{\circ} \mathrm{C} \mathrm{SmC} 72.5{ }^{\circ} \mathrm{C} \mathrm{SmA} 139.5{ }^{\circ} \mathrm{C}$ Ch $143.2{ }^{\circ} \mathrm{C} \mathrm{B} 149.2{ }^{\circ} \mathrm{C} \mathrm{I}$,

- on cooling: I $144.5^{\circ} \mathrm{C} \mathrm{B} 141.5{ }^{\circ} \mathrm{C} \mathrm{Ch}$ $137.7^{\circ} \mathrm{C}$ SmA $70.6{ }^{\circ} \mathrm{C} \mathrm{SmC} 67.8^{\circ} \mathrm{C} \mathrm{SmI}$ $64.7{ }^{\circ} \mathrm{C} \mathrm{SmG} 43.0{ }^{\circ} \mathrm{C} \mathrm{Cr}$.

The X-ray studies were performed on X'Perrt PRO (PANalytical) diffractometer using the $\mathrm{CuK} \alpha$ radiation $(\lambda=1.540589 \AA)$ and a graphite monochromator. The sample was placed in a nickel-plated copper sample holder of dimensions $18 \times 9 \times 0.2 \mathrm{~mm}^{3}$. The temperature of the sample was stabilized (within accuracy $\pm 0.1^{\circ} \mathrm{C}$ ) with a continuous flow cryostat supplied by Anton Paar Co. The sample was not oriented. Prior to the temperature measurements the sample was melted, next cooled to room temperature with the rate of $10 \mathrm{deg} / \mathrm{min}$, and next left for 24 hours at this temperature. After this relaxing period the sample was cooled down to $\mathrm{LN}_{2}\left(-195.5{ }^{\circ} \mathrm{C}\right)$ temperature with a rate of $10 \mathrm{deg} / \mathrm{min}$, and finally left for 12 hours. The patterns were obtained at rising temperature. After each heating stage the sample was allowed to equilibrate for about $10 \mathrm{~min}$. To analyse and fit the spectra, the profile-fitting program FULLPROF based on Rietveld method was used.
Fig. 3 presents an exemplary fit for the two layer thicknesses for the orthorhombic PMMM structure (at a temperature of $40{ }^{\circ} \mathrm{C}$ ). Anyhow, one should keep in mind that PMMM structure should not be identified with the structure of the liquid crystalline phase.

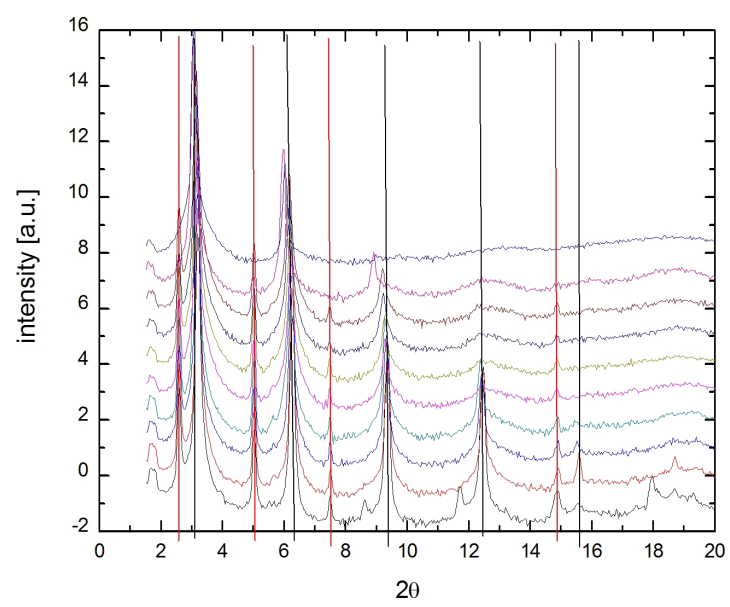

Fig. 3. Fit1 (red lines) and fit 2 (black lines) corresponding to the parameters $c_{1}$ and $c_{2}$.

The $\mathrm{c}_{1}$ parameter is almost stable in the temperature range of $40{ }^{\circ} \mathrm{C}$ to $65^{\circ} \mathrm{C}$. Above $65^{\circ} \mathrm{C}$ up to $70{ }^{\circ} \mathrm{C}$ there are small fluctuations in its value (about $0.25 \AA$ ). And then, at $80^{\circ} \mathrm{C}$ the $\mathrm{c}_{1}$ parameter decreases rapidly (up to $33.75 \AA$ ). For the temperature range of 40 to $50{ }^{\circ} \mathrm{C}$, the $\mathrm{c}_{2}$ parameter is set to be $28.75 \AA$. Between $60{ }^{\circ} \mathrm{C}$ and $70{ }^{\circ} \mathrm{C}$ the $\mathrm{c}_{2}$ parameter increases to $29.25 \AA$.

Minor changes in the $\mathrm{c}$ parameters may suggest dense layers, almost solid-like LC. The changes occur in the vicinity of the phase transitions (to the smectic $C^{*}$ and next to the smectic A phase). From the calculations, the size of the molecules is known (molecule length: $27.1 \AA$, width: $5.0 \AA$ ). Knowing 
the thickness of the layers, we may define the ratio of the thickness to the length of the molecule. The ratios (for fit 1 and fit 2 ) are collected in Table 2 [28].

Table 2. The thickness to length ratio of the molecule for fit 1 and fit 2 within two temperature intervals of CE8.

\begin{tabular}{ccc}
\hline & $65^{\circ} \mathrm{C}-70{ }^{\circ} \mathrm{C}$ & $70{ }^{\circ} \mathrm{C}-80{ }^{\circ} \mathrm{C}$ \\
\hline \hline$\frac{c_{1}}{l}$ & $1.3(22)$ & $1.0(63)$ \\
$\frac{c_{2}}{l}$ & $1.22(7)$ & $1.0(45)$ \\
\hline
\end{tabular}

In the smectic A phase, the layer thickness is approximately equal to the length of the molecule (ratio fit1: 1.0(63) and ratio fit2: 1.0(45)). Thus, we may determine what type of smectic A phase is formed: $A_{1}, A_{2}, A_{1 f}, A_{d}$. We suggest that the substance is either a monolayer smectic A or pseudoferroelectric smectic $\mathrm{A}_{1 \mathrm{f}}$. However, taking into account that we are dealing with the chiral system, it is most likely that we observe smectic $\mathrm{A}_{1 \mathrm{f}}$ [28].

Dielectric measurements were performed at $\mathrm{KU}$ Leuven (Belgium) using a high-resolution dielectric analyser (Alpha Analyzer, Novocontrol Technologies) in the frequency range of $10^{-1}$ up to $10^{6} \mathrm{~Hz}$ [30]. The sample was prepared in a form of a parallel plate capacitor with a dielectric layer thickness of $800 \mathrm{~nm}$. Subsequent dielectric measurements were done at the Institute of Molecular Physics in Poznań (Poland) using HP set-up in the frequency range of $10^{-2}$ to $1.510^{6} \mathrm{~Hz}$. LINKAM $5 \mu \mathrm{m}$ cells were used as the capacitor. These cells were initially calibrated. The sample was oriented by the surface effect producing a planar alignment.

\section{Results and discussion}

A good starting point to perform dielectric spectrum analysis is to separate the data into two main ranges of measuring field: low-frequency and highfrequency dielectric data.

\subsection{Low-frequency dielectric data}

The temperature dependence of $\varepsilon^{\prime}=\varepsilon_{\text {stat }}$ provides information about the measured substance. Conventionally, the response of a medium to static electric fields is described by the low-frequency $(\omega \rightarrow 0)$ limit of permittivity. For liquid crystals, frequencies below $1 \mathrm{kHz}$ could be considered as "static ones". The permittivity relates to a material ability to resist an electric field. One can treat the temperature dependence of $\varepsilon^{\prime}=\varepsilon_{\text {stat }}$ as a classic thermogram. The dielectric and the calorimetric data together prove phase transition points.

Let us analyze the graph shown in Fig. 4. Convergence of dielectric and calorimetric data is clearly visible. All mesophase transition points are present. Additionally, a new transition point (a "dielectric" transition) at $86{ }^{\circ} \mathrm{C}$ occurs (both thicknesses) [30]. As one may see, with going towards SmC phase, $\varepsilon^{\prime}\left(=\varepsilon_{\text {stat }}\right)$ increases, and just after transition to the $\mathrm{SmA}$, drops rapidly, indicating the "dielectric" transition point. Such behavior is typical of chiral molecules, where the ordering short range interaction effect is much stronger than the thermal fluctuations.

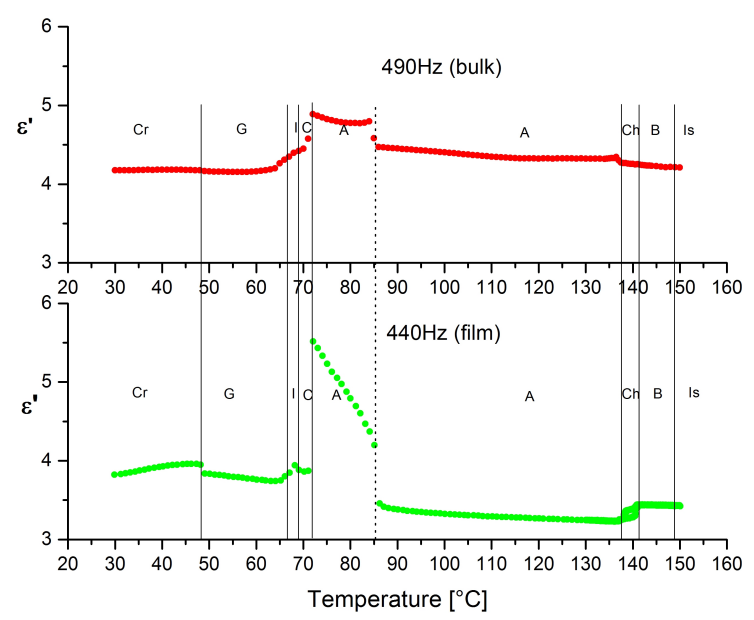

Fig. 4. Electric permittivity $\left(\varepsilon^{\prime}\right)$ measured at the low frequency of $440 \mathrm{~Hz}$ (film $5 \mu \mathrm{m}$ ) and of $490 \mathrm{~Hz}$ (bulk $800 \mu \mathrm{m}$ ). Cr means a crystal state, symbols G, I, C, A stand for SmG, SmI, SmC and SmA phases, respectively, $\mathrm{Ch}$ is cholesteric phase and symbol B represents blue phase region; Is means an isotropic state. Solid lines indicate transition temperature obtained from calorimetric measurement. Dash line indicates transition temperature obtained from dielectric experiment.

Fig. 5 presents the electric permittivity hysteresis loop measured at the low frequency $(440 \mathrm{~Hz})$, 
observed only in the case of thin sample. Hysteresis occurs when the response of a physical system to an external perturbation depends on both the current input and on the previous history of the system. The hysteresis loop may suggest that CE8 liquid crystal have the ability to be easy addressing system. In Fig. 5, the pre-transition region to the blue can be also seen.

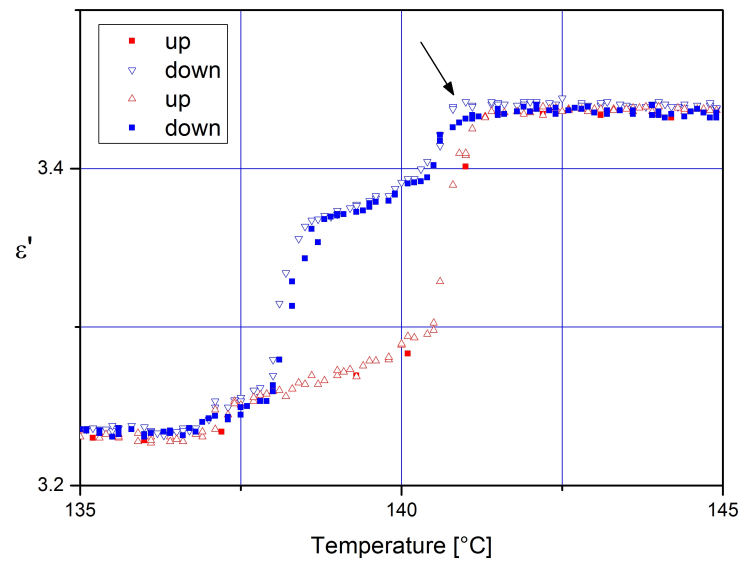

Fig. 5. Permittivity hysteresis loop measured at the low frequency of $440 \mathrm{~Hz}$ for film sample (two runs). Transition to the cholesteric phase is clearly visible. The arrow indicates transition temperature region to the blue phase.

\subsection{High-frequency dielectric data}

For real systems there may be any number of contributions (so-called modes) to the electric permittivity. Assuming that each mode may "relax" at sufficiently different frequencies and the relaxation processes contribute additively to the permittivity, we analysed the data using Cole-Cole (CC) equation:

$$
\varepsilon^{*}(\omega)=\varepsilon_{\infty}^{\prime}+\sum_{k} \frac{\Delta \varepsilon_{k}^{\prime}}{1+\left(i \omega \tau_{k}\right)^{\alpha_{k}}}
$$

where $\Delta \varepsilon_{\mathrm{k}}^{\prime}$ is "an amplitude" (an increment) for each relaxation, $\omega$ is the angular frequency, $\varepsilon_{\infty}^{\prime}$ is the high frequency limit of the dielectric constant, $\tau_{\mathrm{k}}$ is the mean relaxation time $\left(\omega_{\mathrm{k}}=\tau_{\mathrm{k}}^{-1}\right)$ and $\alpha_{\mathrm{k}}$ is the symmetric distribution parameter. The dynamic situation of investigated system is described by Cole-Cole parameters.
We performed measurements on heating and cooling. Fig. 6 presents the 3D plot of data collected on cooling. Fig. 7 presents the dielectric dispersion and absorption data observed at $83{ }^{\circ} \mathrm{C}$.

Assuming a single molecular dipole relaxation as the effective response of the investigated system to an alternating field of frequency $\omega$, we have decomposed the dielectric spectra (observed on cooling) into a sum of Cole-Cole terms (Fig. 8). At low frequencies and for high temperatures, where the Ohmic conduction plays an important role, we evaluated conductivity $\sigma$ (Fig. 9). To extract these specific data we excluded the conductivity contribution from the complex dielectric constants $\varepsilon^{*}$. An elegant way to remove Ohmic conduction from measured loss spectra uses Kramers-Kronig relation, whereby we transformed the real part $\varepsilon^{\prime}$ into an imaginary part $\varepsilon_{\mathrm{KK}}^{\prime \prime}$ that is solely based on relaxation phenomena. By virtue of this, $\varepsilon_{\mathrm{KK}}^{\prime \prime}$ lacks the Ohmic conduction term [31]. Therefore, in turn, it indicates that the resulting process is of molecular origin. Fortunately, for all Cole-Cole terms, parameter $\alpha$ is relatively small (varying from 0.75 to 1.00) (Fig. 10). Due to the small $\alpha$ value it turns out that we are dealing with (almost) Debye-like processes. It seems that molecular interpretation via relaxation time may be more accurate.

\subsection{Interpretation of the molecular mech- anisms.}

We have identified four modes whose parameters are summarized in the Table 3 and their molecular interpretation is given below.

It is shown that almost all observed processes are characterized by $\alpha$ parameter practically equal to 1, typical of Debye processes (Fig. 10). Only the first process for some mesophases seems to be the complex one ( $\alpha$ parameter changing from 0.75 to $0.95)$. In real systems, local fluctuations of molecular structure or environment (the long-range dipolar correlation), which are responsible for a "dielectric friction", might result in a distribution of relaxation times. However, we should note that the translational motion may reduce distribution of relaxation times [9]. Therefore, one might expect Debye-like relaxation in liquid crystals. 

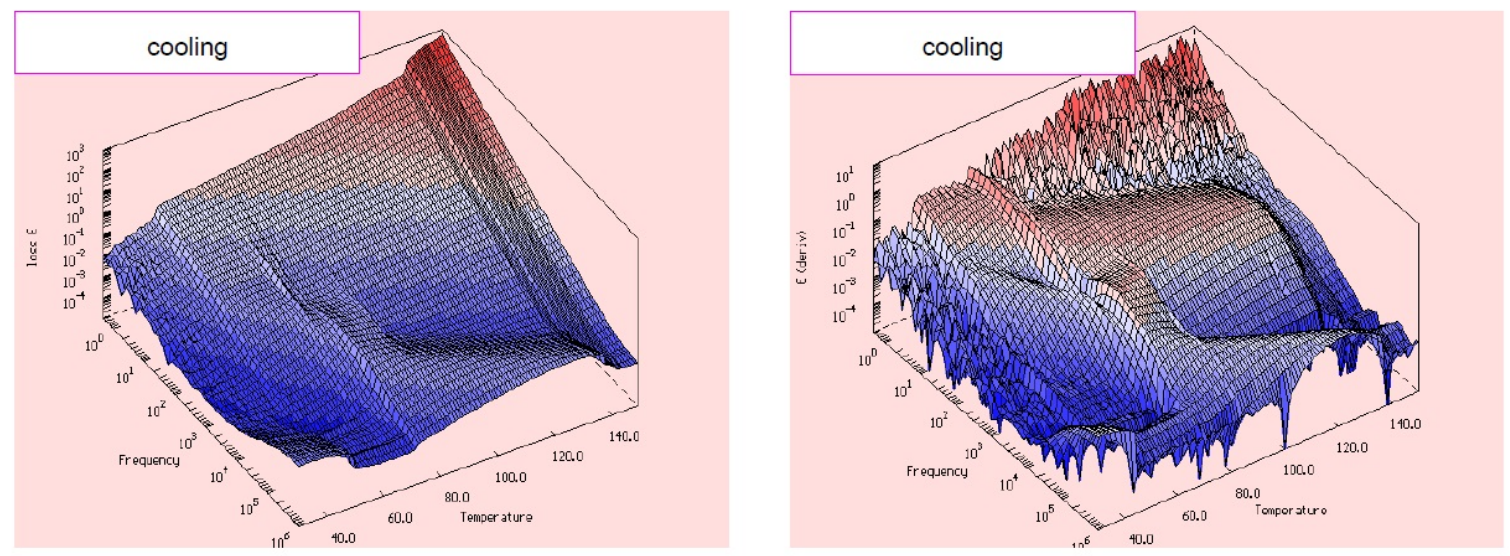

Fig. 6. The dielectric absorption data observed on cooling for CE8. Ordinate axes show the dielectric absorption (left) and derivative of absorption (right).

Table 3. Parameters of identified modes.

\begin{tabular}{|c|c|c|c|c|}
\hline mode & $\alpha_{\mathrm{CC}}$ & $\Delta \varepsilon$ & $\begin{array}{c}\tau \\
{[\mathrm{s}]}\end{array}$ & $\begin{array}{c}\mathrm{E}_{\mathrm{a}} \\
{[\mathrm{kJ} / \mathrm{mol}]}\end{array}$ \\
\hline 1 & $0.75-0.95$ & $\begin{array}{c}5.5-0.9 \\
\left(66^{\circ} \mathrm{C}-132^{\circ} \mathrm{C}\right)\end{array}$ & $\begin{array}{c}53010^{-3}\left(44^{\circ} \mathrm{C}\right) \\
44010^{-6}\left(137^{\circ} \mathrm{C}\right)\end{array}$ & $\begin{array}{l}27.6\left(47^{\circ} \mathrm{C}-65^{\circ} \mathrm{C}\right) \\
24.5\left(74{ }^{\circ} \mathrm{C}-84^{\circ} \mathrm{C}\right) \\
21.5\left(95^{\circ} \mathrm{C}-136^{\circ} \mathrm{C}\right)\end{array}$ \\
\hline 2 & 0.95 & $\begin{array}{c}0.13-0.22 \\
\left(47^{\circ} \mathrm{C}-72^{\circ} \mathrm{C}\right) \\
0.89-1.05 \\
\left(72^{\circ} \mathrm{C}-86^{\circ} \mathrm{C}\right)\end{array}$ & $\begin{array}{l}2710^{-3}\left(44^{\circ} \mathrm{C}\right) \\
4510^{-6}\left(85^{\circ} \mathrm{C}\right)\end{array}$ & $\begin{array}{c}77.5\left(47^{\circ} \mathrm{C}-66^{\circ} \mathrm{C}\right) \\
115.6\left(67{ }^{\circ} \mathrm{C}-72^{\circ} \mathrm{C}\right)\end{array}$ \\
\hline 3 & $0.90-1.00$ & 0.13 & $\begin{array}{l}6510^{-6}\left(68^{\circ} \mathrm{C}\right) \\
2.410^{-6}\left(85^{\circ} \mathrm{C}\right)\end{array}$ & $\begin{array}{l}73.6\left(65^{\circ} \mathrm{C}-86^{\circ} \mathrm{C}\right) \\
37.1\left(86^{\circ} \mathrm{C}-138^{\circ} \mathrm{C}\right)\end{array}$ \\
\hline 4 & 0.80 & 0.10 & $\begin{array}{l}79010^{-6}\left(137^{\circ} \mathrm{C}\right) \\
34010^{-6}\left(150^{\circ} \mathrm{C}\right)\end{array}$ & 27.6 \\
\hline
\end{tabular}

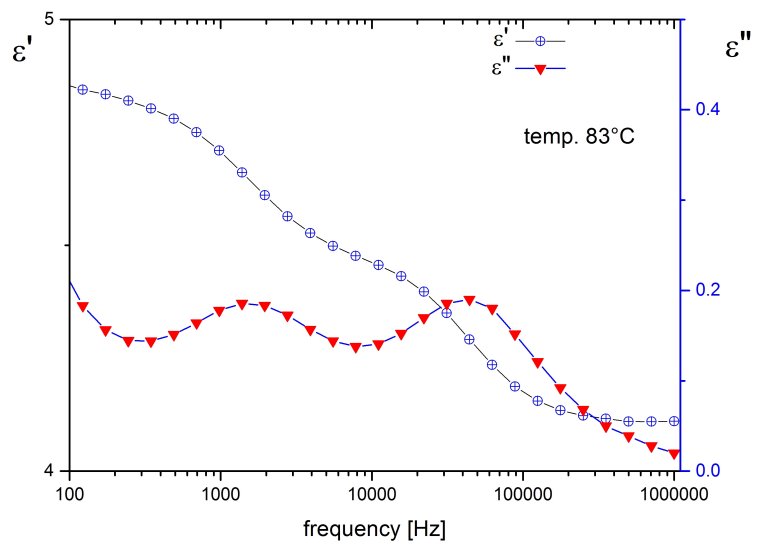

Fig. 7. The dielectric dispersion and absorption data observed at $83{ }^{\circ} \mathrm{C}$.
Nordio-Rigatti-Segre (NRS) gave the theory of relaxation in the complex dielectric permittivity at the local anisotropic potential in a liquid crystal state [32]. NRS theory relates the complex permittivity $\varepsilon^{*}$ to the dipole moment components $\mu_{1}$ and $\mu_{\mathrm{t}}$ of the mesogen. Because the effective dipole moment is normalized with respect to the freemolecule dipole moment, it is clear that finally the authors receive the four different relaxation modes $\left(\mathrm{M}_{\mathrm{pq}} \mathrm{p}, \mathrm{q}=0.1\right)$ : two for the homeotropic orientation $\left(\mathrm{M}_{00}\right.$ and $\left.\mathrm{M}_{01}\right)$ and two for the planar one $\left(\mathrm{M}_{10}\right.$ and $\left.\mathrm{M}_{11}\right)$ [9]. Each component of the effective dipole moment contains two contributions from the molecular dipole moment, and each 


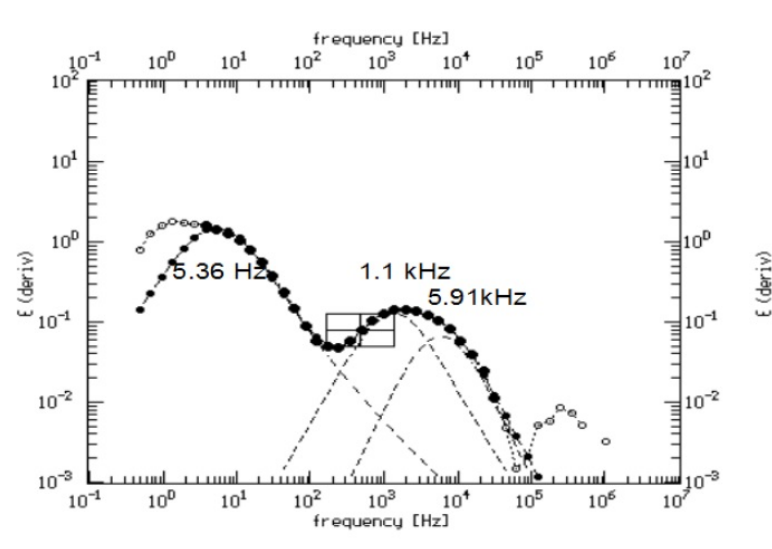

Fig. 8. The complex spectra decomposition graph at $70{ }^{\circ} \mathrm{C}$. The ordinate shows the derivative of absorption $\left(\Delta \varepsilon_{1}=2.47, \alpha_{1}=0.9, \mathrm{f}_{1}=5.36 \mathrm{~Hz}\right.$, $\Delta \varepsilon_{2}=0.19, \alpha_{2}=0.95, \mathrm{f}_{2}=1.08 \mathrm{kHz}, \Delta \varepsilon_{3}=$ $\left.0.1, \alpha_{3}=0.99, \mathrm{f}_{3}=5.91 \mathrm{kHz}\right)$.

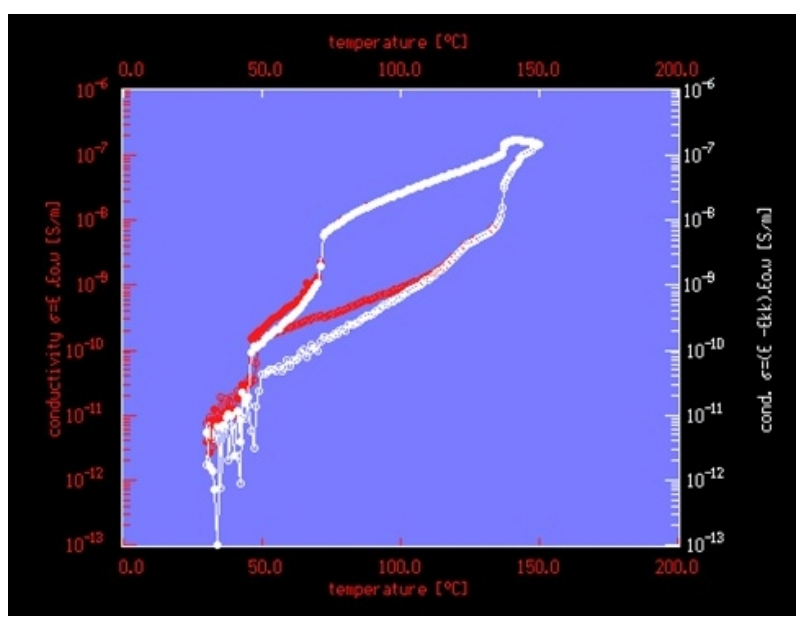

Fig. 9. The conductivity $\sigma$ (log scale) calculated from complex spectra on heating and cooling. The left ordinate shows $\sigma=\varepsilon_{0} \varepsilon^{\prime \prime} \omega$. The right ordinate shows $\sigma=\varepsilon_{0}\left(\varepsilon^{\prime \prime}-\varepsilon_{\mathrm{KK}}\right) \omega$, where $\varepsilon_{\mathrm{KK}}$ is calculated from Kramers-Kronig equation.

can have different relaxation time. However, for an uniaxial molecule with two independent moments of inertia, the dynamics can be approximately described in terms of three different relaxation times (or frequencies) [9]. Ferrarini et al. [33] have evaluated numerically NRS theory. They have confirmed the existence of three different relaxation time modes. The first one results from end-overend rotation. This mode is strongly dependent upon the order parameter (in other words this mode is

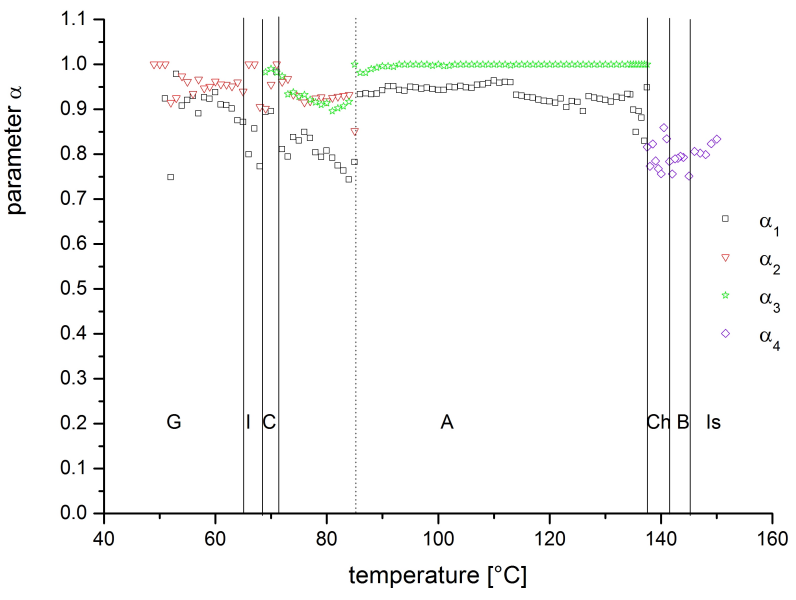

Fig. 10. The parameter $\alpha$ calculated from the complex spectra on cooling. Symbols G, I, C, A stand for SmG, SmI, SmC and SmA phases, respectively, $\mathrm{Ch}$ is cholesteric phase and symbol B represents blue phase region; Is means isotropic state. Solid lines indicate transition temperature obtained from calorimetric measurement. Dash line indicates transition temperature obtained from the dielectric experiment [30].

strongly temperature dependent). It is characterized by a long time scale. The second one is rotation about its own long molecular axis. It is characterized by an average time scale. The third one is a processional motion about director. It is characterized by a very short time scale.

The electric permittivity $\left(\varepsilon^{\prime}\right)$ (on cooling) remains almost independent of temperature in the cholesteric, blue and isotropic phases. Thus, the static structure of dipoles is undisturbed by phase transitions and almost unchanged from the isotropic phase to the blue phase.

Discussed process is of Cole-Cole type throughout isotropic, blue and cholesteric phases. Parameter $\alpha$ is different from one $(\langle\alpha\rangle=0.8)$, suggesting the superposition of processes (more than one relaxation time). We didn't apply any external ordering agent to CE8 sample. Thus, symmetric distribution of the relaxation time (Cole-Cole process) is a natural consequence of "bulk" dielectric measurements. According to the Nordio-Rigatti-Segre theory, in the isotropic phase, one might observe two main processes; 
reorientations around the long and short molecular axes. Since the transverse dipole moment components have a little value, and the dielectric amplitude (proportional to the dipole $\Delta \varepsilon \propto \mu^{2}$ ) is relatively small $(\Delta \varepsilon \approx 0.1)$, we assume that molecular origin of the process is reorientation around the long molecular axes. The time distribution is due to temperature fluctuation of the long molecular axes. The relaxation time $\tau$ is of the order of millisecond $\left(0.34 \mathrm{~ms}\right.$ at $\left.150{ }^{\circ} \mathrm{C}\right)$ and gives an average value of the activation energy as $27.6 \mathrm{~kJ} / \mathrm{mol}$. For comparison, the activation energy of 4,4'-di-heptyloxyazoxybenzene 7.OAOB in isotropic phase $E_{\text {iso }}=27 \mathrm{~kJ} / \mathrm{mol}$. This value is reasonable, because the freedom of rotation of the long molecular axis of a bulk sample is limited. The second process is invisible. This may lead to the conclusion that the longitudinal components of the dipole moments exhibit antiparallel correlation in the isotropic phase. The dielectric amplitude does not show any significant change at the phase transition points. As a result, we suggest that we are dealing with the same process at the blue and cholesteric phases. In the blue phase, where the regular three-dimensional cubic structure appears, the activation energy is unchanged. It seems that the molecular dynamics stabilizes this phase. The freedom of reorientations around the long molecular axis in a twisted and bulk structure is similar. The relaxation time is $0.79 \mathrm{~ms}$ at $137{ }^{\circ} \mathrm{C}$. Thus, the activation energy of $27.6 \mathrm{~kJ} / \mathrm{mol}$ is reasonable in these phases (for instance, for 7.OAOB in nematic phase $\mathrm{E}_{\text {long }}=$ $24 \mathrm{~kJ} / \mathrm{mol}$ ). While cooling down to the cholesteric phase, we didn't observe any significant change in the molecular parameters. It means that the cholesteric phase is a stable dynamic structure. We suggest that coupling between chirality and the dipole-dipole interaction of electric dipoles of two short axes might induce a biaxial helix. Such a structure can stabilize double-twisted structures.

While cooling towards smectic phase, we observe that the molecular dynamics significantly changes. The conductivity calculated from complex spectra (on cooling) shows well defined steps. The first step (at $136{ }^{\circ} \mathrm{C}$ ) is typical of a first order phase transition (Fig. 9) and indicates cholesteric (chiral nematic) to smectic transition. At low temperatures, the change in conductivity is due to the formation of nuclei, typical of crystallization. The second step (at $71{ }^{\circ} \mathrm{C}$ ) is typical of a first order phase transition, as well. The third step (at $43{ }^{\circ} \mathrm{C}$ ) indicates crystallization.

On cooling we have decomposed the dielectric spectrum into two main processes.

The first process has a relatively big dielectric amplitude $\Delta \varepsilon=1.0$ to 5.5. Because the total dipole $\left(\mu_{\text {tot }}=2.27 \mathrm{D}\right)$ is almost parallel to the long molecular axis $\left(\beta=164.2^{\circ}\right)$, we suggest that molecular origin of this process is the reorientation around the short molecular axis (end-over-end rotation). The process is slow what might be due to the dense molecular packing, observed, for instance, in X-ray experiment [28].

Time varies with temperature from $440 \mu$ s (at $136{ }^{\circ} \mathrm{C}$ ) to $530 \mathrm{~ms}$ (at $44{ }^{\circ} \mathrm{C}$ ). Ferroelectric liquid crystal (D)-2-(2-bromo-3-methyl-butanoyloxy)6-(4-n-decyloxybenzoyloxy)-naphthalene $(\mathrm{Br} 4)$ (which is almost a rod-like molecule with the length of $36.5 \AA$ and the width of $8.5 \AA$ ) has the total dipole $\left(\mu_{\text {tot }}=3.30 \mathrm{D}\right)$ almost parallel to the long molecular axis $\left(\mu_{1}=2.7 \mathrm{D}\right)$. For this substance we have observed similar process. We assigned it to the reorientation around the short molecular axis [34].

Mean activation energy (calculated from the Arrhenius plot) is $21.5 \mathrm{~kJ} / \mathrm{mol}$ (from $136{ }^{\circ} \mathrm{C}$ to $95^{\circ} \mathrm{C}$ ), $24.5 \mathrm{~kJ} / \mathrm{mol}$ (from $84^{\circ} \mathrm{C}$ to $71{ }^{\circ} \mathrm{C}$ ) and $27.6 \mathrm{~kJ} / \mathrm{mol}$ (from $65{ }^{\circ} \mathrm{C}$ to $47{ }^{\circ} \mathrm{C}$ ) (Fig. 11). The increase in energy values with decreasing temperature is due to the crystallization. The process is of Cole-Cole type. The smallest value of the parameter $\alpha$ is 0.75 . However, in the smectic phase we are dealing with (almost) single time Debye-like process $(\alpha=0.95)$.

Second process, visible in the "liquid-like" smectic phases is the fastest. Relaxation time is changing from $69 \mathrm{~ns}$ (at $136{ }^{\circ} \mathrm{C}$ ) to $65 \mu \mathrm{s}$ (at $68{ }^{\circ} \mathrm{C}$ ). The relaxation strength (dielectric amplitude) is relatively small $(\Delta \varepsilon=0.13)$ and temperature independent. Through all phases, the process has been found to be the activation process. Up to 


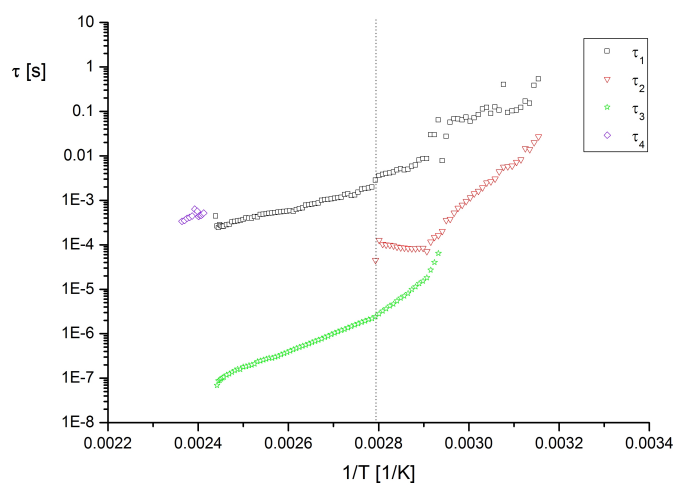

Fig. 11. Arrhenius plot calculated from complex spectra on cooling. Symbols G, I, C, A stand for SmG, SmI, SmC and SmA phases, respectively, $\mathrm{Ch}$ is a cholesteric phase and symbol B represents blue phase region; Is means isotropic state. Dash line indicates transition temperature obtained from the dielectric experiment.

$86{ }^{\circ} \mathrm{C}$ the activation energy is $37.1 \mathrm{~kJ} / \mathrm{mol}$ : the process is single time Debye-like $(\alpha=1.0)$. The activation energy of about $40 \mathrm{~kJ} / \mathrm{mol}$ for the observed reorientation, is proper for instance, for bananalike liquid crystals [35]. In the temperature range of $86{ }^{\circ} \mathrm{C}$ to $65^{\circ} \mathrm{C}$ the activation energy is $73.6 \mathrm{~kJ} / \mathrm{mol}$ : the process is (almost) single time Debye-like $(\alpha \propto 0.9$ to 1.0$)$.

We assume that the foregoing process may be due to the conformational motion (intra reorientation) of the CE8 molecules. We know from the calculations for an isolated molecule (using the quantum chemical program MOPAC/7, with the AM1 Hamiltonian) [36] that the CE8 molecule can exist in many different conformations. Thus, conformational changes as the interpretation of molecular dynamics are likely. At the phase transition, the SmC-SmI process is completely frozen.

While going towards $\mathrm{SmC}$ phase at $86{ }^{\circ} \mathrm{C}$, we notice an abrupt change in the electric permittivity $\left(\varepsilon^{\prime}=\varepsilon_{\text {stat }}\right)$, visible for both "film" and "bulk" samples. The electric permittivity increases (which is particularly evident for "film" sample). This means that the effective dipole moment increases. Therefore, we suggest formation of dimmers (molecular pairs), resulting in parallel (ferroelectric) order in the short range. For parallel arrangement we expect strong positive dipole-dipole correlation. This is consistent with the suggestion of the formation of nuclei at $\mathrm{N}^{*}$-SmA transition. The small curvatures in the curves of time $\left(\tau_{1}\right.$ and $\left.\tau_{3}\right)$ observed at $86{ }^{\circ} \mathrm{C}$ (Fig. 11) are due to the appearance of dimmer structure. From dynamic point of view we observe the third molecular motion which is almost the Debye-like ( $\alpha \approx 0.95)$ process. Till the transition to $\mathrm{SmC}$, this process is not thermally activated. Molecularly, we interpret this process as a linear response of statistically oriented ferroelectric clusters. The amplitude (the relaxation strength) varies from 1.05 at $86^{\circ} \mathrm{C}$ to 0.89 at $72{ }^{\circ} \mathrm{C}$. Relaxation time is $45 \mu$ s at $85^{\circ} \mathrm{C}$. Above the SmASmC phase transition, the static and dynamic situations are subject to change. The electric permittivity $\left(\varepsilon^{\prime}=\varepsilon_{\text {stat }}\right)$ is decreasing going towards transition to SmI phase (for both thin and bulk samples: Fig. 5). Therefore, we suggest formation of anti-parallel order in the short range. A way to describe the strength of this effect is to introduce the dipole-dipole correlations g-factor. Because of lack of some molecular data, we cannot calculate the g-factor for the CE8 molecule. However, one may take into consideration a g-factor for a similar substance, namely (4'-hexyloxybenzoate) 4(2-methylobuthyl) phenol (CE6). Fig. 12 presents temperature dependence of the anisotropic dipoledipole correlation factor $g$ for parallel and perpendicular components of the dipole moment of CE6 in smectic and nematic phases [37]. As shown, the correlation as a function of temperature can vary from parallel to antiparallel arrangement. One may compare the presented g dipole-dipole correlation factor to the Kirkwood correlation factor given by Hudge et al. [38].

Below transition to SmG phase the electric permittivity $\left(\varepsilon^{\prime}=\varepsilon_{\text {stat }}\right)$ becomes constant up to crystal phase.

Molecular motion is now the activation process. From $72{ }^{\circ} \mathrm{C}$ to $67^{\circ} \mathrm{C}$, the activation energy is $115.6 \mathrm{~kJ} / \mathrm{mol}$. From $66^{\circ} \mathrm{C}$ to $47^{\circ} \mathrm{C}$ the activation energy is $77.5 \mathrm{~kJ} / \mathrm{mol}$. The change in relaxation strength (dielectric amplitude) is relatively small, from 0.22 to 0.13 in the temperature range from $72{ }^{\circ} \mathrm{C}$ to $47{ }^{\circ} \mathrm{C}$. Molecular origin of this (almost) Debye-like $(\alpha \approx 0.95)$ process is reorientation of 


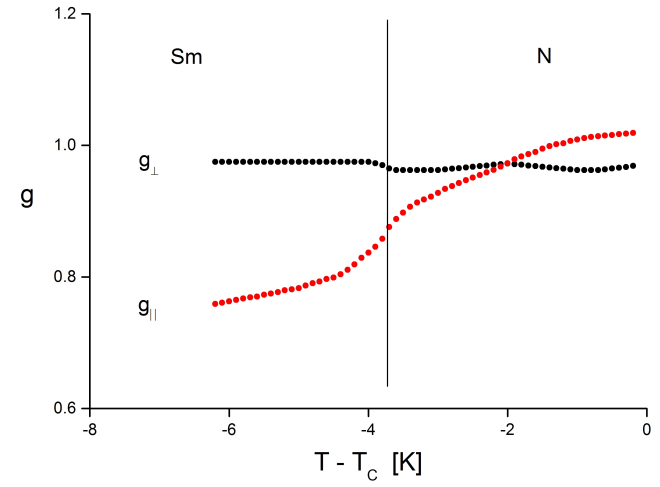

Fig. 12. Temperature dependence of g-factor obtained for CE6 (4-(2-methylbutyl)phenyl-4'hexyloxybenzoate) for the smectic and nematic phases [30].

the rigid core around the long molecular axis. Relaxation time is $27 \mathrm{~ms}$ at $44{ }^{\circ} \mathrm{C}$.

\section{Conclusions}

For 4-(2-methylbytyl)phenyl 4-(4octylphenyl)benzoate we observe complex molecular dynamics in all mesophases, including the blue one. The analysis of the dielectric relaxation processes allows us to make a general statement: we deal with Debye-like or almost Debye-like molecular processes. The origin of observed processes is reorientation of the rigid core about the short and long molecular axes as well as intra reorientation. We have found the existence of dipole-dipole interaction observed by the formation of ferroelectric clusters. Introducing chirality to a nematic rod-like molecule means the formation of helicity (the helical axis is formed) along the short axis (in the chiral nematic phase). A rod-like molecule may have two distinguishable axes. Coupling between chirality and the two short axes may induce a biaxial helix (independent helix along each of the short axes). Such a structure is able to stabilize double-twisted BP phase. Our compound has two different short axes. We suggest the possibility of existence of a double-helix structure stabilizing the blue phase.

\section{Acknowledgements}

Special thanks to all who helped and inspired Authors; especially to Professors W. Kuczyński, J. Hoffmann and M.
Wübbenhorst and as well as Bram Vanroy for help in measurements and valuable discussions.

\section{References}

[1] Coles H.J., Pivnenko M.N., Nature, 436 (2005), 997.

[2] Yamamoto J., Nishiyama I., Inoue M., YOKOYAMA H., Nature, 437 (2005), 525.

[3] Kikuchi H., Yokota M., Hisakado Y., Yang H., KaJiYAma T., Nat. Mater., 1 (2002), 64.

[4] Ge Z., Gauza S., Jiao M., Xianyu H., Wu S.T., Appl. Phys. Lett., 94 (2009), 101104.

[5] Rao L., Ge Z., Wu S.T., LeE S.H., Appl. Phys. Lett., 95 (2009), 231101.

[6] Rao L., Yan J., Wu S.T., Yamamoto S., Haseba Y., Appl. Phys. Lett., 98 (8) (2011), 081109.

[7] Lee H., Park H.J., Kwon O.J., Yun S.J., PArk J.H., Hong S., Shin S.T., SID Int. Symp. Dig. Tec., 42 (2011), 122.

[8] Meiboom S., Sethna J.P., Anderson W.P., Brinkman W.F., Phys. Rev. Lett., 46 (1981), 1216.

[9] ОтоWsKi W., Molecular motion in liquid crystals as seen via dielectric spectroscopy, in: GALEWSKI Z., SOBCZYK L. (Eds.), Dielectric Properties of Liquid Crystals, Transworld Research Network, Kerala, India, 2007, pp. $1-35$.

[10] Cava R.J., Patel J.S., Rietman E.A, J. Appl. Phys., 60 (1986), 3093.

[11] DEMUS D., private information.

[12] Hoffmann J., Acta Phys. Pol. A, 105 (2004), 373.

[13] KuCzyński W., Stegemeyer H., Liq. Cryst., 5 (2) (1989), 553

[14] Gierlotka S., Przedmojski J., Pura B., Liq. Cryst., 3 (11) (1988), 1535.

[15] Raszewski Z., RutKowska J., KedZierski J., Perkowski P., PieceK W., Zieliński J., ZMiJA J., R. Dąbrowski R., Mol. Cryst. Liq. Cryst., 263 (1) (1995), 271.

[16] Przedmojski J., Dąbrowski R., Pura B., Zicket K., GierlotKa S., Mol. Cryst. Liq. Cryst., 151 (1987), 171.

[17] Przedmojski J., Gierlotka S., WASniewski R. Pura B., ZAJĄC W., Ferroelectrics, 92 (1989), 345.

[18] Budai J., Pindak R., Davey S.C, Goodby J.W., J. Physique Lett., 45, (1984), 9.

[19] KutnjaK Z., Ferroelectrics, 369 (1) (2008), 133.

[20] Bone H.F., Coates D., Davey A.B., Mol. Cryst. Liq. Cryst, 102 (10) (1984), 331.

[21] Walton H.G, Coles H.J., Ferroelectrics, 147 (1) (1993), 223.

[22] Drevensex I., Musevic I., Copic M., Phys. Rev. A, 41 (1990), 923.

[23] KuCZyński W., Phys. Rev. E, 81 (2010), 021708.

[24] Kutnjak Z., Kralu S., Zumer S., Phys. Rev. E, 66 (2002), 041702.

[25] Kutnjak Z., Cordoyiannis G., Nounesis G., Ferroelectrics, 294 (1) (2003), 105. 
[26] Rosenzweig J.D, Collings P.J., Phys. Rev. E, 47 (1993), 1876.

[27] Lewińska G., Otowski W., Acta Phys. Pol. A, 117 (4) (2010), 562.

[28] LEWIŃsKa G., PhD Thesis, Institute of Nuclear Physics Polish Academy of Sciences, Krakow, Poland (2012).

[29] GALEWSKI Z., private information.

[30] Отоwski W., Lewińska G., Acta Phys. Pol. A, 125 (5) (2014), 1152.

[31] Wübbenhorst M., Van Turnhout J., J. NonCryst. Solids, 305 (2002), 40.

[32] Nordio P.L., Rigatti G., Segre U., Mol. Phys. 25 (1973), 129.

[33] Ferrarini A., Nordio P.L., Moro G.J., Diffusion Models for Molecular Motion in Uniaxial Mesophases, in: Luckhurst G.R., Veracini C.A. (Eds.), The Molecular Dynamics of Liquid Crystals, NATO ASI Series C, 431 (1994), pp. $41-69$.
[34] Otowski W., González Y., Palacios B., Da la Fuente M.R., PÉrez Jubindo M.A., Ferroelectrics, 180 (1996), 93.

[35] Otowski W., Budziak A., Dobrowolska A., Mol. Cryst. Liq. Cryst., 494 (2008), 1.

[36] Отоwski W., Demus D., Mol. Cryst. Liq. Cryst., 366 (2001), 533.

[37] Otowski W., Demol W., Van Dael W., Mol. Cryst. Liq. Cryst., 226 (1993), 103.

[38] Hudge P.G., Lokhande M.P. And KumbHarkhane A.C., Indian J. Phys., 86 (9) (2012), 813.

Received 2014-10-13 Accepted 2015-01-22 